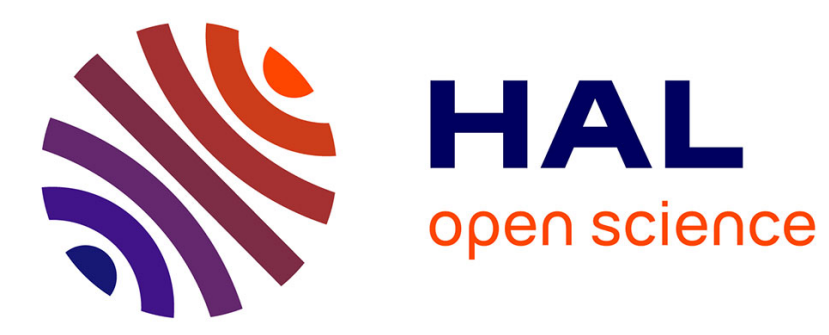

\title{
Fault diagnosis on a magnetically supported plate
}

\author{
Lothar Kiltz, Mamadou Mboup, Joachim Rudolph
}

\section{To cite this version:}

Lothar Kiltz, Mamadou Mboup, Joachim Rudolph. Fault diagnosis on a magnetically supported plate. 1st International Conference Systems and Computer Science, Aug 2012, Lille, France. hal-00756109

\section{HAL Id: hal-00756109 \\ https://hal.inria.fr/hal-00756109}

Submitted on 22 Nov 2012

HAL is a multi-disciplinary open access archive for the deposit and dissemination of scientific research documents, whether they are published or not. The documents may come from teaching and research institutions in France or abroad, or from public or private research centers.
L'archive ouverte pluridisciplinaire HAL, est destinée au dépôt et à la diffusion de documents scientifiques de niveau recherche, publiés ou non, émanant des établissements d'enseignement et de recherche français ou étrangers, des laboratoires publics ou privés. 


\title{
Fault diagnosis on a magnetically supported plate
}

\author{
Lothar Kiltz*, Mamadou Mboup ${ }^{\dagger}$, and Joachim Rudolph* \\ ${ }^{*}$ Chair of Systems Theory and Control Engineering, Saarland University, Email: \{1.kiltz,j.rudolph $\} @ 1$ lsr.uni-saarland.de \\ ${ }^{\dagger}$ CReSTIC - UFR SEN, Université de Reims Champagne-Ardenne, Email: mamadou.mboup@univ-reims.fr
}

\begin{abstract}
A model-based online fault-diagnosis scheme for an electromagnetically supported plate is presented as an example of a nonlinear and open-loop unstable system. First, residuals for sensor as well as for actuator faults are generated using algebraic derivative estimators. Then, the robust detection and isolation of step-like sensor and actuator faults is presented. The performance of the proposed algorithms is illustrated by experimental results.
\end{abstract} tion.

Index Terms-Diagnosis, Derivative estimation, Change detec-

\section{INTRODUCTION}

The improvement of safety, reliability, and performance of industrial processes has become an important problem in automatic control. In that context, fault diagnosis including fault detection and isolation (FDI) is a key challenge.

In this article, a model-based FDI method is applied to an experimental setup. The diagnosis system relies on the concept of analytical redundancy, i. e., measured behaviour of the process is compared to its nominal behaviour, where the latter is defined by a mathematical model. Deviations between measured and nominal behaviour are evaluated to detect and isolate a faulty component.

During the previous two decades, many approaches for linear systems have been well established for theoretical cases. Examples are approaches based on observers including Kalman filters [1], parity relations [2], parameter estimation [3], [4], or statistical tests [5], respectively. Useful surveys can be found in the excellent books [6], [7], and [8]. For nonlinear systems, great effort is typically required for the realization of a model-based FDI system (cf. [9] and the geometric approach), and particularly, when accounting for model uncertainties (cf. for example recent works [10], [11]). This effort may be eased using so-called algebraic techniques as demonstrated in [12], [13], and [14].

These results motivate the new algebraic diagnosis scheme presented here for abrupt sensor and actuator faults on a magnetically supported plate. FDI on this type of system is particularly challenging as it exhibits fast dynamics and dominant nonlinearities. Good performance of the proposed approach has been demonstrated in a real-time implementation.

This article is organized as follows. Mathematical models of the physical system, the closed-loop controller, and the considered faults are stated in section II. The mathematical models are used in section III to derive a diagnosis scheme consisting of the generation of residuals (section III-A) and their processing (section III-C). The real-time calculation of the residuals is facilitated by derivative estimation techniques explained in section III-B. Experimental results are shown in section IV.

\section{DESCRIPTION OF THE SYSTEM}

A suitable mathematical model plays a key rôle in the fault diagnosis approach presented here. Therefore, the experimental setup, its mathematical model, and the discussed faults are explained in this section.

\section{A. Experimental setup}

The experimental setup consists of a rectangular aluminium plate with four iron profiles at its corners. Four electromagnets mounted to the frame above the iron profiles allow for the application of attracting forces to lift the plate. The vertical displacement between the plate and the frame is measured by three sensors.

The currents through the magnet coils are driven by current controllers thus allowing the coil currents to be considered as the control inputs. The dynamics of the magnetic field generation are neglected due to the fact that the iron cores of the electromagnets and the iron profiles at the plate are laminated to prevent the generation of eddy currents. Furthermore, the magnetic forces are assumed to pull on the centers of the iron profiles, since only small tilt angles are achieveable. Under these assumptions, the plate can schematically be drawn as shown in Fig. 1, where $u=\left(u_{1}, \ldots, u_{4}\right)^{T}$ denotes the currents through the magnetic coils, $y=\left(y_{1}, y_{2}, y_{3}\right)^{T}$ denotes the measured displacements between the plate and the frame, and $F_{i}\left(u_{i}, y\right)$ are the magnetic forces. Horizontal translations in the $x_{0}-y_{0}$ plane as well as rotations about the $z_{b}$ direction are neglected. These considerations together with the assumption of the plate being a rigid body simplify the discussion of the plate motions as the basis of the proposed diagnosis system.

\section{B. Mathematical model of the dynamic system}

Under the assumptions stated above, equations of motion describing the vertical translation of the plate and its rotation about the $x_{b}$ and $y_{b}$ axes can be written as

$$
\left(\begin{array}{c}
m\left(\frac{\ddot{y}_{2}+\ddot{y}_{3}}{2}-g\right)+F_{1}+F_{2}+F_{3}+F_{4} \\
k_{1} \frac{\ddot{y}_{2}-\ddot{y}_{1}}{2}-F_{1}+F_{2}-F_{3}+F_{4} \\
k_{2} \frac{\ddot{y}_{3}-\ddot{y}_{1}}{2}-F_{1}-F_{2}+F_{3}+F_{4}
\end{array}\right)=0
$$

with $k_{1}=\frac{J_{x}}{l_{d y} l_{f y}}, k_{2}=\frac{J_{y}}{l_{d x} l_{f x}}$. Here $F=\left(F_{1}, \ldots, F_{4}\right)^{T}$ denotes the magnetic forces, $m, J_{x}$, and $J_{y}$ denote the mass of the plate and its moment of inertia about the $x_{b}$ and $y_{b}$ 


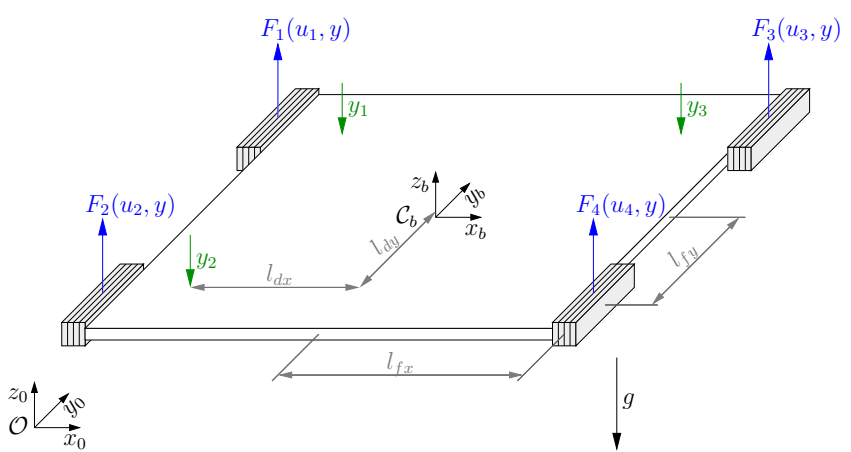

Fig. 1. Schematic of the plate's kinematics.

axes, respectively. Additionally, $l_{f x}$ and $l_{f y}$ are the horizontal distances between the center of mass of the plate and the centers of the iron profiles in the $x_{b}$ and $y_{b}$ directions, respectively, while $l_{d x}$ and $l_{d y}$ determine the positions of the displacement sensors (cf. Fig. 1). The magnetic forces are modelled as

$$
F_{i}=\frac{u_{i}^{2}}{g_{i}\left(h_{i}\right)}, \quad i=1, \ldots, 4,
$$

where $h_{i}=\zeta_{i}(y)$ are the displacements between the magnets and the corresponding iron profiles at the center of the profiles:

$$
\zeta(y)=\frac{1}{2}\left(\begin{array}{ccc}
k_{x}+k_{y} & 1-k_{y} & 1-k_{x} \\
k_{x}-k_{y} & 1+k_{y} & 1-k_{x} \\
-k_{x}+k_{y} & 1-k_{y} & 1+k_{x} \\
-k_{x}-k_{y} & 1+k_{y} & 1+k_{x}
\end{array}\right) y,
$$

with $k_{x}=\frac{l_{f x}}{l_{d x}}, k_{y}=\frac{l_{f y}}{l_{d y}}$, while $g_{i}: \mathbb{R} \rightarrow \mathbb{R}$ approximates the measured relationship between the coil current of the $i$-th magnet, its generated force, and $h_{i}$. The functions $g_{i}$ are nonnegative and strictly monotonically increasing on the relevant range of displacement between magnet and iron profile.

\section{Closed-loop control}

Rewriting the eqs. of motion (1a) as

$$
q:=\left(\begin{array}{l}
F_{1}+F_{2}+F_{3}+F_{4} \\
F_{1}-F_{2}+F_{3}-F_{4} \\
F_{1}+F_{2}-F_{3}-F_{4}
\end{array}\right)=\left(\begin{array}{c}
m\left(g-\frac{\ddot{y}_{2}+\ddot{y}_{3}}{2}\right) \\
k_{1} \frac{\ddot{y}_{2}-\ddot{y}_{1}}{2} \\
k_{2} \frac{\ddot{y}_{3}-\ddot{y}_{1}}{2}
\end{array}\right)
$$

motivates the following flatness-based trajectory tracking controller for the stabilization of the outputs $y_{m, i}, i=1,2,3$, of the sensors measuring $y_{i}$ around desired trajectories $t \mapsto$ $y_{d}(t)=\left(y_{d, 1}(t), \ldots, y_{d, 3}(t)\right)^{T}$ :

$$
\begin{aligned}
q_{d} & =\left(\begin{array}{c}
m\left(g-\frac{v_{2}+v_{3}}{2}\right) \\
k_{1} \frac{v_{2}-v_{1}}{2} \\
k_{2} \frac{v_{3}-v_{1}}{2}
\end{array}\right), \\
v_{i} & =\ddot{y}_{d, i}-l_{i, 1} \dot{e}_{m, i}-l_{i, 2} e_{m, i}-l_{i, 3} \int_{t_{0}}^{t} e_{m, i} \mathrm{~d} \tau,
\end{aligned}
$$

where $q_{d}$ denotes the desired value of $q$, and $e_{m, i}=y_{m, i}-y_{d, i}$. Desired magnetic forces $F_{d}$, minimizing the mean variation
$\sum_{i=1}^{4}\left(F_{d, i}-\frac{1}{4} \sum_{j=1}^{4} F_{d, j}\right)^{2}$, are given by

$$
F_{d}=\frac{1}{4}\left(\begin{array}{cccc}
\frac{-k_{1}-k_{2}}{2} & \frac{-m+k_{1}}{2} & \frac{-m+k_{2}}{2} & m \\
\frac{k_{1}-k_{2}}{2} & \frac{-m-k_{1}}{2} & \frac{-m+k_{2}}{2} & m \\
\frac{-k_{1}+k_{2}}{2} & \frac{-m+k_{1}}{2} & \frac{-m-k_{2}}{2} & m \\
\frac{k_{1}+k_{2}}{2} & \frac{-m-k_{1}}{2} & \frac{-m-k_{2}}{2} & m
\end{array}\right)\left(\begin{array}{c}
v_{1} \\
v_{2} \\
v_{3} \\
g
\end{array}\right) .
$$

Desired values $u_{d}$ of $u$ follow by inverting (1b):

$$
u_{d, i}=\sqrt{F_{d, i} g_{i}\left(\zeta_{i}\left(y_{m}\right)\right)}, \quad i=1, \ldots, 4 .
$$

\section{Mathematical model of the faults}

Both sensor faults $y_{e}=y_{m}-y$ and actuator faults $u_{e}=$ $u_{d}-u$ are considered. The actual magnetic forces following from (1b) and (3d) are

$$
F_{i}=\frac{\left(\sqrt{F_{d, i} g_{i}\left(\zeta_{i}\left(y_{m}\right)\right)}-u_{e, i}\right)^{2}}{g_{i}\left(\zeta_{i}\left(y_{m}-y_{e}\right)\right)} .
$$

Thus, the actual magnetic forces can be written as the sum of desired forces $F_{d}$ and erroneous forces $F_{e}: F=F_{d}+F_{e}$. The components $F_{e, i}, i=1, \ldots, 4$, of $F_{e}$ are given by

$$
F_{e, i}=\frac{g_{e, i} F_{d, i}+u_{e, i}\left(u_{e, i}-2 \sqrt{F_{d, i} g_{i}\left(\zeta_{i}\left(y_{m}\right)\right)}\right)}{g_{i}\left(\zeta_{i}\left(y_{m}-y_{e}\right)\right)},
$$

where $g_{e, i}=g_{i}\left(\zeta_{i}\left(y_{m}\right)\right)-g_{i}\left(\zeta_{i}\left(y_{m}-y_{e}\right)\right)$. That is, a single fault on the $j$-th actuator, $j \in\{1, \ldots, 4\}$, only influences the corresponding magnetic force:

$$
F_{e, i}= \begin{cases}\frac{u_{e, i}\left(u_{e, i}-2 u_{d, i}\right)}{g_{i}\left(\zeta_{i}\left(y_{m}\right)\right)}, & i=j, \\ 0, & i \neq j,\end{cases}
$$

while a single fault on the $j$-th sensor, $j \in\{1,2,3\}$, causes each magnetic force to become erroneously:

$$
F_{e, i}=\left(\frac{g_{i}\left(\zeta_{i}\left(y_{m}\right)\right)}{g_{i}\left(\zeta_{i}\left(y_{m}-y_{e}\right)\right)}-1\right) F_{d, i}, \quad i=1, \ldots, 4 .
$$

Substituting $F=F_{d}+F_{e}$ and (3c) into (1a) yields an expression for the influence of the force errors on $\ddot{y}$ :

$$
\ddot{y}=P F_{e}+v,
$$

where $v=\left(v_{1}, v_{2}, v_{3}\right)^{T}$, and

$$
P=\left(\begin{array}{llll}
p_{1} & p_{2} & p_{3} & p_{4} \\
p_{2} & p_{1} & p_{4} & p_{3} \\
p_{3} & p_{4} & p_{1} & p_{2}
\end{array}\right)
$$

with $p_{1}=-\frac{1}{m}-\frac{1}{k_{1}}-\frac{1}{k_{2}}, p_{2}=-\frac{1}{m}+\frac{1}{k_{1}}-\frac{1}{k_{2}}, p_{3}=$ $-\frac{1}{m}-\frac{1}{k_{1}}+\frac{1}{k_{2}}, p_{4}=-\frac{1}{m}+\frac{1}{k_{1}}+\frac{1}{k_{2}}$.

Remark 1. Since the mathematical model (1) consists of fewer independent equations than the number of independent faults, it is in general not possible to isolate simultaneously occurring faults. Therefore, it will be assumed in the following discussion that at most one fault occurs within a sufficiently short period of time. This assumption is justified by the very unlikely simultaneous failure of two or more components.

Remark 2. Faults of the type $z(t)=\xi \mathrm{H}\left(t-t_{\xi}\right), z \in$ $\left\{u_{e, 1}, \ldots, u_{e, 4}, y_{e, 1}, \ldots, y_{e, 4}\right\}$, with $\xi \in \mathbb{R}$ and $\mathrm{H}$ the Heaviside function, will be of particular interest in the following discussion. They will be called abrupt faults. 


\section{FAUlt Diagnosis}

In principle, the proposed real-time diagnosis works as follows: in a first step, the mathematical model (1) is used to derive residual signals containing information on present faults. The real-time calculation of the residuals is facilitated by algebraic methods for estimating derivatives. Afterwards, the residuals are evaluated to detect and isolate abrupt faults, i. e., to decide whether an abrupt fault has occurred and which component is affected. The residual evaluation is insensitive to slow changes in the residuals.

\section{A. Residual generation}

The eqs. of motion (1a) allow for the expression of each magnetic force with respect to the other system variables:

$$
F=\frac{1}{3}\left(\begin{array}{l}
m g-F_{2}-F_{3}+F_{4} \\
m g-F_{1}+F_{3}-F_{4} \\
m g-F_{1}+F_{2}-F_{4} \\
m g+F_{1}-F_{2}-F_{3}
\end{array}\right)-A \ddot{y}=: \lambda(F, \ddot{y}),
$$

where

$$
A=\frac{1}{6}\left(\begin{array}{ccc}
k_{1}+k_{2} & m-k_{1} & m-k_{2} \\
-k_{1}+k_{2} & m+k_{1} & m-k_{2} \\
k_{1}-k_{2} & m-k_{1} & m+k_{2} \\
-k_{1}-k_{2} & m+k_{1} & m+k_{2}
\end{array}\right) .
$$

Thus, residuals are obtained by $r=\lambda\left(F_{d}, \hat{\ddot{y}}_{m}\right)-F_{d}$, where the approximation $\hat{\ddot{y}}_{m}$ of $\ddot{y}_{m}$ is numerically estimated from $y_{m}$ (cf. section III-B). Since $r$ can be rewritten as

$$
r=B F_{e}-A\left(\ddot{y}_{e}+\tilde{y}_{m}\right),
$$

where $\tilde{y}_{m}=\hat{\ddot{y}}_{m}-\ddot{y}_{m}$, and

$$
B=\frac{1}{3}\left(\begin{array}{cccc}
3 & 1 & 1 & -1 \\
1 & 3 & -1 & 1 \\
1 & -1 & 3 & 1 \\
-1 & 1 & 1 & 3
\end{array}\right)
$$

the residuals are sensitive to actuator faults, sensor faults, and derivative estimation errors.

\section{B. Algebraic estimation of derivatives}

An important component of the residual generation is the real-time estimation of time derivatives from noisy signals. In [15], [16], a method for estimating derivatives in realtime applications is explained using differential algebraic manipulations and Mikusiński's operational calculus [17]. It is shown that this is equivalent to a polynomial approximation of the desired derivative. Since this property of the derivative estimators is of particular importance for the further discussion, relevant key aspects are briefly explained in this section.

1) Basic principle: Assume that an estimate of the $n$ th order time derivative $x^{(n)}$ of a signal $x$ at a point of time $t$ is required. An optimal (in a least-squares sense) linear approximation of the values of $x^{(n)}$ on a time interval $\mathcal{I}_{T, \Delta T}(t)=[t-\Delta T-T, t-\Delta T]$ can be written as [18]

$$
\hat{x}_{T, \Delta T}^{(n)}(t)=\sum_{i=0}^{N} c_{i, T}(t-\Delta T) \varphi_{i}(T+\Delta T),
$$

where $\mathcal{B}=\left\{\varphi_{i}\right\}_{i=0, \ldots, N}$ is a set of basis functions. For $\varphi_{i}, \varphi_{j} \in \mathcal{B}$ being pairwise orthonormal on $[0, T]$ with respect to a weight function $w$, the expansion coefficients $c_{i, T}$ are the weighted projections of $x^{(n)}$ on $\varphi_{i}$ :

$$
c_{i, T}(t)=\int_{0}^{T} w(\tau) \varphi_{i}(\tau) x^{(n)}(t-T+\tau) \mathrm{d} \tau .
$$

To eliminate the unknown values of $x^{(n)}$ in (10), repeatedly integrate by parts to obtain

$$
\begin{aligned}
c_{i, T}(t)= & \sum_{k=0}^{n-1}(-1)^{k}\left[\left(w \varphi_{i}\right)^{(k)}(\tau) x^{(n-1-k)}(t-T+\tau)\right]_{\tau=0}^{T} \\
& +(-1)^{n} \int_{0}^{T}\left(w \varphi_{i}\right)^{(n)}(\tau) x(t-T+\tau) \mathrm{d} \tau .
\end{aligned}
$$

with $\left(w \varphi_{i}\right)(\tau)=w(\tau) \varphi_{i}(\tau)$. The boundary values are eliminated by $w(\tau)=(T-\tau)^{\alpha} \tau^{\beta}, \alpha, \beta>n-1$. This determines $\mathcal{B}$ to be a set of Jacobi polynomials [19]. In the following discussion, it will be assumed that $\varphi_{i}$ denotes the corresponding Jacobi polynomial of degree $i$.

Remark 3. When $x^{(n)}$ is analytic, the approximation $\hat{x}_{T, \Delta T}^{(n)}$ is a $N$-th degree truncated Taylor series expansion of $x^{(n)}$, and the approximation error $\tilde{x}^{(n)}=\hat{x}_{T, \Delta T}^{(n)}-x^{(n)}$ is given by $\tilde{x}^{(n)}=-\sum_{i=N+1}^{\infty} c_{i, T}(t-\Delta T) \varphi_{i}(T+\Delta T)$. Hence, it is reasonable to choose $T+\Delta T$ to be a root of $\varphi_{N+1}$ if a delayed derivative estimation is tolerable [15], [16].

Remark 4. This estimation method implicitly reduces the influence of measurement noise [16]. Therefore, the influence of noise on the diagnosis system is not discussed in this article.

2) Real-time implementation: Combining (9) and (11), the required derivative estimation is given by

$$
\hat{x}_{T, \Delta T}^{(n)}(t)=\int_{0}^{T} h(\tau) x(t-\Delta T-T+\tau) \mathrm{d} \tau,
$$

with the kernel $h(\tau)=\sum_{i=0}^{N}(-1)^{n}\left(w \varphi_{i}\right)^{(n)}(\tau) \varphi_{i}(T+$ $\Delta t$ ) being independent of $t$. An approximation of (12) can efficiently be implemented in a real-time application by a time-discrete finite impulse response filter $\hat{x}_{L, \Delta t}^{(n)}[k]=$ $\sum_{j=0}^{L} \bar{h}_{j} x[k-L+j]$ with constant weights $\left(\bar{h}_{0}, \ldots, \bar{h}_{L}\right)$.

3) Abruptly changing derivatives: When $x^{(n)}$ can be decomposed into a continous part $x_{c}^{(n)}$ and a step: $x^{(n)}(t)=$ $x_{c}^{(n)}(t)+\xi \mathrm{H}\left(t-t_{\xi}\right)$, the output of the derivative estimator is given by $\hat{x}_{T, \Delta T}^{(n)}(t)=\hat{x}_{c, T, \Delta T}^{(n)}(t)+\xi \psi\left(t-t_{\xi}-\Delta T\right)$, where

$$
\begin{aligned}
\psi(t) & = \begin{cases}0, & t<0, \\
\sum_{j=0}^{N} \kappa_{j}(t), & 0 \leq t<T, \\
1, & T \leq t,\end{cases} \\
\kappa_{j}(t) & =\varphi_{j}(T+\Delta T) \int_{T-t}^{T}\left(w \varphi_{j}\right)(\tau) \mathrm{d} \tau .
\end{aligned}
$$

Hence, the output of $\hat{x}_{T, \Delta T}^{(n)}$ changes continuously from $\hat{x}_{c, T, \Delta T}^{(n)}$ to $\hat{x}_{c, T, \Delta T}^{(n)}+\xi$ during $\left[t_{\xi}+\Delta T, t_{\xi}+\Delta T+T\right]$, and 
the estimation error $\tilde{x}^{(n)}=\hat{x}_{T, \Delta T}^{(n)}-x^{(n)}$ reads as

$$
\tilde{x}^{(n)}(t)=\tilde{x}_{c}^{(n)}(t)+\xi\left(\psi\left(t-t_{\xi}-\Delta T\right)-\mathrm{H}\left(t-t_{\xi}\right)\right) .
$$

\section{Residual evaluation}

Each residual in (8) is affected by every fault. Furthermore, the residuals cannot be expected to converge to zero even in the fault-free case in the practical application due to model uncertainties. Therefore, processing of available signals is explained in this section that facilitates the fast detection and isolation of abrupt faults (cf. remark 2) on sensors and actuators. The proposed method shows robustness with respect to measurement noise and model uncertainties.

1) Sensor faults: Consider an abrupt fault on the $i$-th sensor: $y_{m, i}(t)=y_{i}(t)+\xi \mathrm{H}\left(t-t_{\xi}\right)$. Let $\hat{y}_{i}$ denote an estimate of $y_{m, i}$ obtained by (9). If $\Delta T>0$, i.e., $\hat{y}_{i}$ is extrapolated from past measurements, the approximation error $\tilde{y}_{i}=\hat{y}_{i}-y_{m, i}$ satisfies

$$
\forall t \in\left[t_{\xi}, t_{\xi}+\Delta T\right]: \tilde{y}_{i}(t)=\tilde{y}_{i, c}(t)-\xi,
$$

where $\tilde{y}_{i, c}=\hat{y}_{i}-y_{i}$, due to (14) and (13). If $T$ and $\Delta T$ are sufficiently small, $\tilde{y}_{i, c}$ can approximately be neglected in (15), and $\tilde{y}_{i}$ is suitable to detect and isolate abrupt sensor faults without a delay by comparing $\left|\tilde{y}_{i}\right|$ with a threshold.

Remark 5. The immediate detection and compensation of discontinuities in the sensor signals is crucial to make the reliable calculation of the residuals possible, since an approximation of the derivative of a discontinuous signal via low order polynomials does not result in reasonable values. In addition, abrupt sensor faults are diagnosed completely independent of actuator faults, which makes the detection and isolation of the latter from the residuals easier.

2) Actuator faults: In the case of a single abrupt fault on the $i$-th actuator, (7) can be decomposed into continuous parts and steps: $\ddot{y}_{m}(t)=\ddot{y}_{c}(t)+\bar{\xi} \mathrm{H}\left(t-t_{\xi}\right)$, where

$$
\begin{aligned}
\ddot{y}_{c}(t) & =v(t)+P_{i}\left(F_{e, i}(t)-\bar{F}_{e, i}\right) \mathrm{H}\left(t-t_{\xi}\right), \\
\bar{\xi} & =P_{i} \bar{F}_{e, i},
\end{aligned}
$$

with $\bar{F}_{e, i}=F_{e, i}\left(t_{\xi}\right)$, and $P_{i}$ the $i$-th column of $P$. Using (14), the estimation error is given by

$$
\tilde{\ddot{y}}_{m}(t)=\tilde{\ddot{y}}_{c}(t)+P_{i} \bar{F}_{e, i}\left(\psi\left(t-t_{\xi}-\Delta T\right)-H\left(t-t_{\xi}\right)\right) .
$$

Since $A P=-B$, the residuals (8) can be written as

$$
\begin{aligned}
r(t) & =B_{i} \nu(t)-A \tilde{\ddot{y}}_{c}(t), \\
\nu(t) & =F_{e, i}(t)-\bar{F}_{e, i}\left(H\left(t-t_{\xi}\right)-\psi\left(t-t_{\xi}-\Delta T\right)\right),
\end{aligned}
$$

where $B_{i}$ denotes the $i$-th column of $B$.

When $T$ is sufficiently small, $F_{e, i}$ can be approximated by a constant $\bar{F}_{e, i, t}$ during each interval $[t+\Delta T, t+\Delta T+T]$, and an approximation of (17) is given by

$$
\hat{r}(t)=B_{i} \bar{F}_{e, i, t} \psi\left(t-t_{\xi}-\Delta T\right)-A \tilde{\ddot{y}}_{c}(t)
$$

with $\psi$ according to (13). That is, the evolution of the residuals in case of an abrupt fault of height $\bar{F}_{e, i}$ at $t_{\xi}$ is a continous transition from 0 to $\bar{F}_{e, i}$ during $\left[t_{\xi}+\Delta T, t_{\xi}+\Delta T+T\right]$, which is scaled by $B_{i}$.

Remark 6 . The analysis of the approximation error $\hat{r}-r$ will be regarded elsewhere.

In the practical application, only corrupted residuals $r_{m}=$ $r+r_{e}$ are available for evaluation, where $r_{e}$ is due to model uncertainties. Therefore, $r_{e}$ has to be suppressed to allow for reliable and sensitive detection and isolation of abrupt actuator faults. In [20], a framework is given for designing suitable finite impulse response filters

$$
\Pi\left[r_{m}\right](t)=\int_{0}^{T_{\Pi}} g(\tau) r_{m}\left(t-T_{\Pi}+\tau\right) \mathrm{d} \tau
$$

using differential algebraic manipulations and Mikusiński's operational calculus [17]. Since the temporal behaviour of such filters is of particular interest in the further discussion, their design and properties in the time domain will be explained in the scope of the current problem.

For $T_{\Pi}>T, \Delta T<0$, and $T_{\Pi}$ and $T$ being sufficiently small, the filter output can be approximated using (18) as

$$
\begin{aligned}
\hat{\Pi}\left[r_{m}\right](t)= & B_{i} \bar{F}_{e, i, t} \chi\left(t-t_{\xi}-\Delta T\right)-A \Pi\left[\tilde{\ddot{y}}_{c}\right](t) \\
& +\bar{r}_{e, t}\left(G\left(T_{\Pi}\right)-G(0)\right),
\end{aligned}
$$

where $G(t)=\int_{0}^{t} g(\tau) \mathrm{d} \tau$, and

$$
\begin{aligned}
& \chi(t)= \begin{cases}0, & t<0, \\
\eta_{T_{\Pi}}^{T_{\Pi}}(t), & 0 \leq t<T, \\
\eta_{T_{\Pi}-t}^{T_{\Pi}-t}(t), & T \leq t<T_{\Pi}, \\
\eta_{0}^{T_{\Pi}+T-t}(t), & T_{\Pi} \leq t<T_{\Pi}+T, \\
G\left(T_{\Pi}\right)-G(0), & T_{\Pi}+T \leq t,\end{cases} \\
& \eta_{a}^{b}(t)=\int_{a}^{b} g(\tau) \psi\left(t-T_{\Pi}+\tau\right) \mathrm{d} \tau+G\left(T_{\Pi}\right)-G(b) .
\end{aligned}
$$

Therefore, the filter design can be interpreted as the choice of a continuous, piecewise differentiable function $\left[0, T_{\Pi}\right] \ni t \mapsto$ $G(t)$ with $G(0)=G\left(T_{\Pi}\right)=0$ that eliminates $\bar{r}_{e, t}$ in (20):

$$
\hat{\Pi}\left[r_{m}\right](t)=B_{i} \bar{F}_{e, i, t} \chi\left(t-t_{\xi}-\Delta T\right)-A \Pi\left[\tilde{\ddot{y}}_{c}\right](t) .
$$

That is related to the kernel in (19) by $g=\frac{\mathrm{d} G}{\mathrm{~d} t}$.

Therefore, faults can be detected by comparing each component of $\left|\Pi\left[r_{m}\right]\right|$ with a threshold. Due to the structure of $B$, the component of $\Pi\left[r_{m}\right]$ corresponding to the faulty actuator is excited most: if the $i$-th actuator is faulty, then $\left|\Pi\left[r_{m, j}\right]\right|=\frac{1}{3}\left|\Pi\left[r_{m, i}\right]\right|$ for $j \neq i$. Thus, the corresponding component of $\Pi\left[r_{m}\right]$ crosses the threshold faster than the other components. This fact is used for fault isolation.

\section{EXPERIMENTAL RESULTS}

In this section, experimental results obtained using the proposed algorithms are given for two scenarios: an abrupt fault on the first sensor (section IV-A) and an abrupt fault on the first actuator (section IV-B). In both cases, the plate is intended to hover horizontally oriented at $z_{0}=-1.5 \mathrm{~mm}$, i. e., the desired values of the measured positions are $y_{i, d}=1.5 \mathrm{~mm}$ for $i=1,2,3$. 


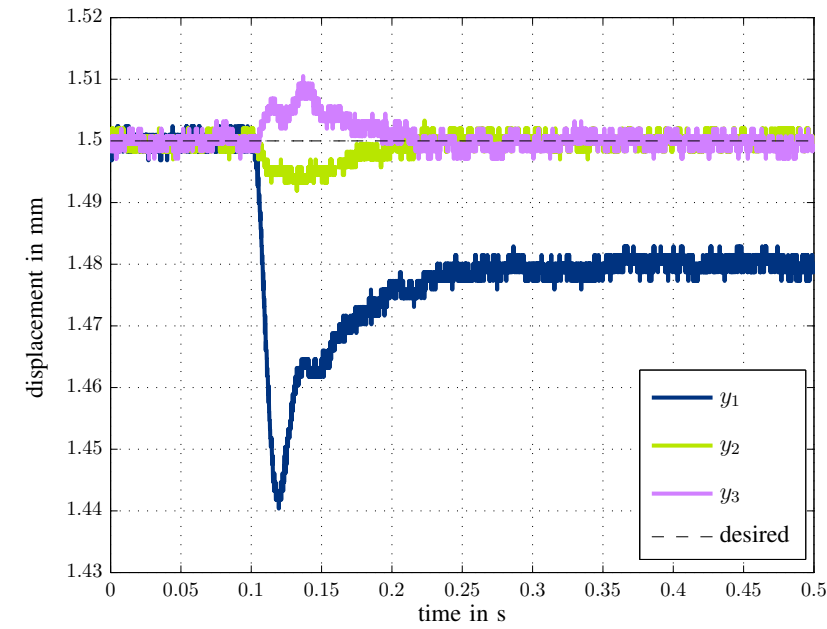

(a)

Fig. 2. Abrupt fault on the first sensor. (a) Actual displacements.

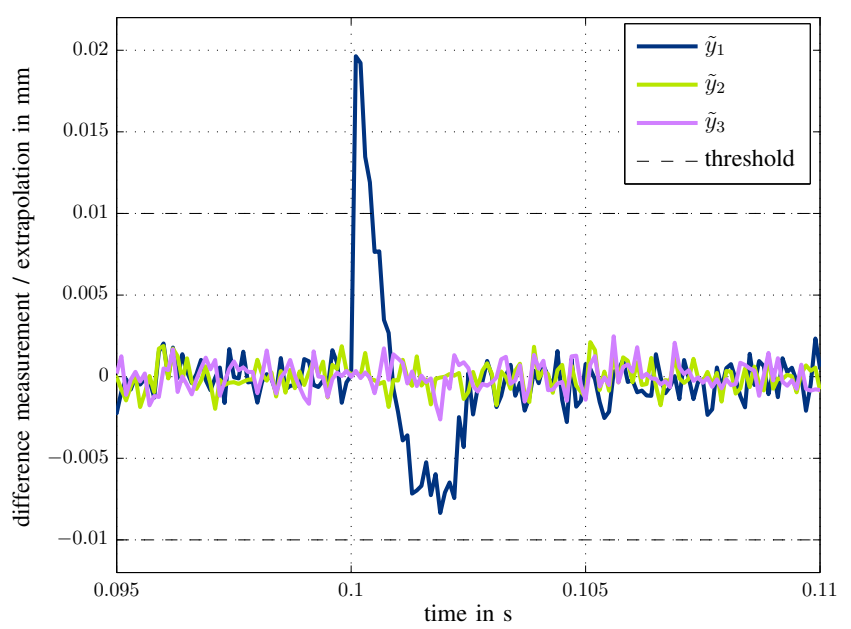

(b)

(b) Differences between measured and extrapolated displacements.

Remark 7. The control hardware of type Indel INFO-SAM2 is based on an $800 \mathrm{MHz}$ PowerPC 750 FX 32 Bit processor, which runs the proprietary real-time operating system INOS. Both closed loop control and fault diagnosis are executed at a sampling period of $100 \mu \mathrm{s}$. The finite impulse response filters used for sensor fault detection, for derivative estimation, and for actuator fault detection, respectively, operate on 25,121 , and 163 samples, respectively. This takes a calculation time of $15 \mu \mathrm{s}$ for fault diagnosis in each sampling step.

\section{A. Sensor fault}

Fig. 2a shows actual displacements $y_{1}, y_{2}$, and $y_{3}$, where the first sensor becomes abruptly faulty by $20 \mu \mathrm{m}$ at $t=0.1 \mathrm{~s}$. The controller is able to restabilize the measured displacement $y_{1, m}$ at $1.5 \mathrm{~mm}$, but clearly the actual displacement does not match its intended value any longer due to the sensor fault. The corresponding evolution of the sensor fault signals $\tilde{y}_{i}$, $i=1,2,3$, according to section III-C2 is shown in Fig. 2 b. Here, the fault on the first sensor is clearly visible as a peak in $\tilde{y}_{1}$ in the sampling step immediately after the fault has occurred. Additionally, neither $\tilde{y}_{2}$ nor $\tilde{y}_{3}$ are visibly affected by the fault even if $y_{2}$ and $y_{3}$ are affected by it temporarily. Therefore, the fault can realibly be detected and isolated in the sampling step after its occurence.

\section{B. Actuator fault}

Fig. 3a shows actual displacements $y_{1}, y_{2}$, and $y_{3}$, where the first actuator becomes abruptly faulty by $100 \mathrm{~mA}$ (about $7 \%$ of $\left.u_{d, 1}\right)$ at $t=0.1 \mathrm{~s}$. As a consequence of the abrupt loss of the force of the first magnet, the plate is tilted. Thereafter, the position of the plate and its orientation are restabilized by the closed-loop control to the desired values. The residuals according to section III-A are depicted in Fig. 3b. As expected, each residual is nonzero in the fault-free case due to model uncertainties. Additionally, each residual is affected by the fault. However, $r_{m, 1}$ (corresponding to the abrupt fault on the first actuator) is affected most. The outputs of the change detection filters described in section III-C2 are presented in Fig. 3c. Here, it can be seen that each filter responds to the fault, with $\Pi\left[r_{m, 1}\right]$ being most affected. A more detailed view on the relevant step detection filter responses is shown in Fig. $3 \mathrm{~d}$. It can be seen that $\Pi\left[r_{m, 1}\right]$ reaches the threshold earlier than the outputs of the other step detection filters. Therefore, a realible detection and isolation of abrupt actuator faults can be reached by applying the proposed FDI scheme. The delay of $6.8 \mathrm{~ms}$ between fault occurrence and fault detection is roughly equivalent to the fastest time constant of $7.3 \mathrm{~ms}$ of the error dynamics of the closed control loop.

\section{CONClusion}

In this article, a method for model-based online fault diagnosis applied to an experimental setup has been explained. Abrupt sensor and actuator faults were considered. Residuals are generated directly from the mathematical model of the physical system using numerically estimated derivatives. Reduction of measurement noise is obtained by estimating derivatives via weighted integration of measured signals. To obtain robustness with respect to slowly varying model uncertainties, signals are post-processed by finite impulse response filters that detect abrupt changes. The algorithms have been implemented on the real-time hardware and their expected properties have been confirmed by experimental results.

\section{ACKNOWLEDGMENT}

The presented work has been funded by the French C.N.R.S. in the PEPS project "Algèbre différentielle et calcul opérationnel pour la SUReté de Foncionnement: Evaluation des Résidus" (SURFER). The authors would like to thank Cédric Join for most useful discussions.

\section{REFERENCES}

[1] P. M. Frank, "Fault diagnosis of dynamic systems using analytical and knowledge-based redundancy - A survey and some new results," Automatica, vol. 26, pp. 459-474, 1990. 


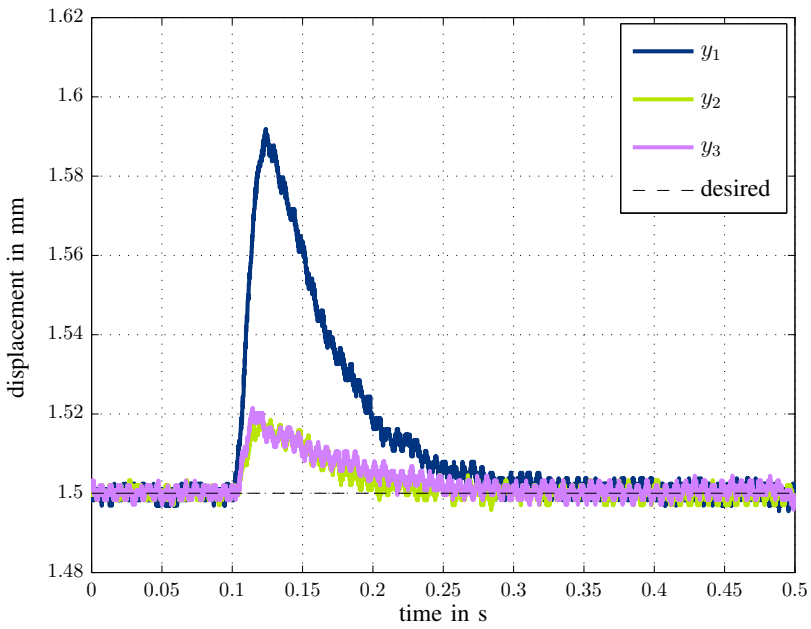

(a)

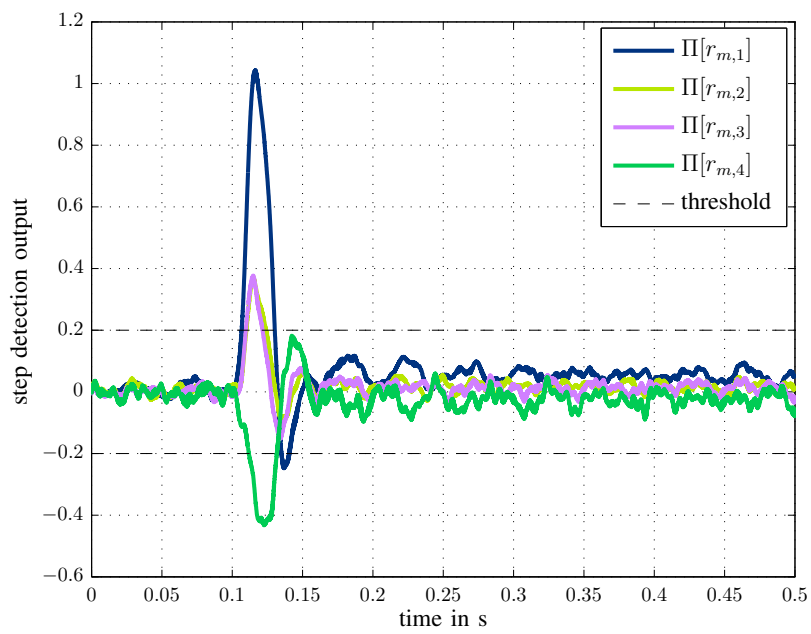

(c)

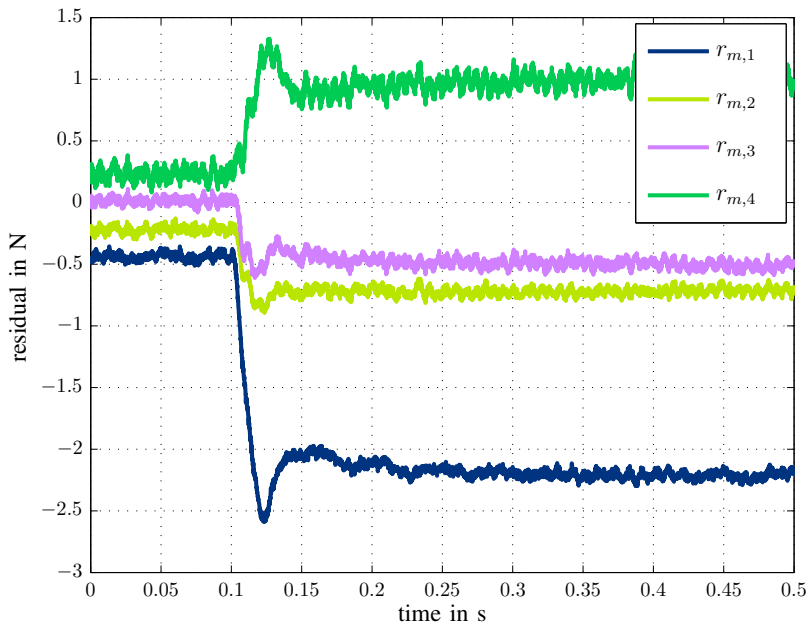

(b)

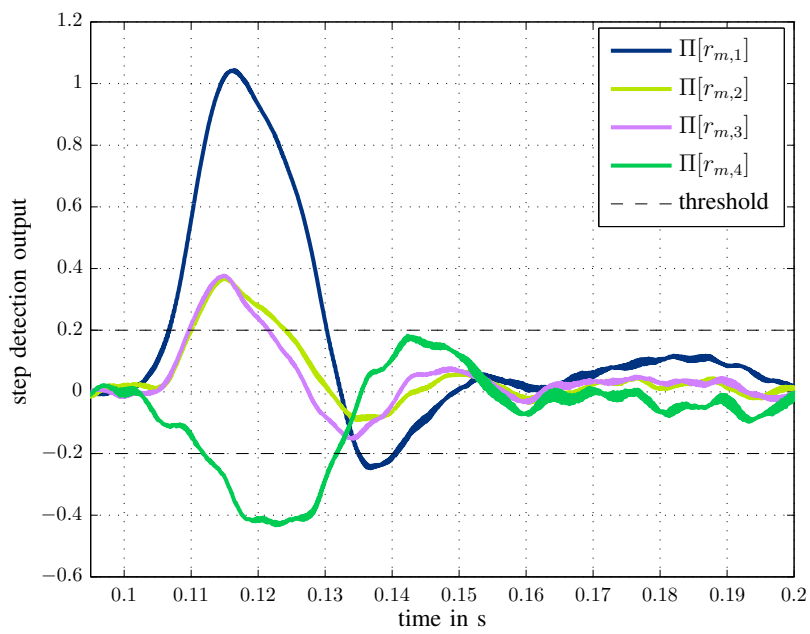

(d)

Fig. 3. Abrupt fault on the first actuator. (a) Displacements. (b) Residuals. (c) Output of the step detection filters. (d) Output of the step detection filters (detail).

[2] J. Gertler and D. Singer, "A new structural framework for parity equation based failure detection and isolation," Automatica, vol. 26, pp. 381-388, 1990.

[3] R. J. Patton, P. M. Frank, and R. N. Clark, Issues of fault diagnosis for dynamic systems. Springer, London, 2000.

[4] R. Isermann, "Fault-detection and fault diagnosis methods - An introduction," Control Engineering Practice, vol. 5, pp. 639-652, 1997.

[5] M. Basseville and V. Nikiforov, Detection of abrupt changes: Theory and application. Prentice Hall, Englewood Cliffs, NJ, 1993.

[6] J. Gertler, Fault detection and diagnosis in engineering systems. Marcel Dekker, New York, 1998.

[7] J. Chen and R. J. Patton, Robust model-based fault diagnosis for dynamic systems. Kluwer Academic Publishers, Boston, MA, USA, 1999.

[8] M. Blanke, M. Kinnaert, J. Lunze, and M. Staroswiecki, Diagnosis and fault-tolerant control. Springer, Berlin, 2003.

[9] C. De Persis and A. Isidori, "A geometric approach to nonlinear fault detection and isolation," IEEE Transactions on Automatic Control, vol. 46, pp. 853-865, 2001.

[10] X. Zhang, M. M. Polycarpou, and T. Parisini, "Fault diagnosis of a class of uncertain nonlinear systems with Lipschitz nonlinearities," in Fault Detection, Supervision and Safety of Technical Processes, Barcelona, Spain, 062010.

[11] S. M. M. Alavi and M. Saif, "A decentralized technique for robust simultaneous fault detection and control of uncertain systems," in American Control Conference, Baltimore, MD, USA, 062010.
[12] M. Fliess, C. Join, and H. Sira-Ramirez, "Nonlinear estimation is easy," International Journal of Modeling, Identification and Control, vol. 4, pp. 12-27, 2008.

[13] P. Mai and C. Hillermeier, "Fault tolerant tracking control for nonlinear systems based on derivative estimation," in American Control Conference, Baltimore, MD, USA, 062010.

[14] A. M. Ali, C. Join, and F. Hamelin, "A robust algebraic approach to fault diagnosis of uncertain linear systems," in Conference on Decision and Control and European Control Conference, Orlando, Florida, USA, 122011.

[15] M. Mboup, C. Join, and M. Fliess, "A revised look at numerical differentiation with an application to nonlinear feedback control," in The 15th Mediterrean Conference on Control and Automation - MED'2007, Athènes Greece, 062007.

[16] —, "Numerical differentiation with annihilators in noisy environment," Numerical Algorithms, vol. 50, pp. 439-467, 2009.

[17] J. Mikusiński, Operational Calculus. Pergamon Press, 1959.

[18] R. Courant and D. Hilbert, Methoden der mathematischen Physik Bd. 1. Julius Springer, 1924

[19] G. Szegö, Orthogonal polynomials. American Mathematical Society, 1959.

[20] M. Fliess, C. Join, and M. Mboup, "Algebraic change-point detection," Applicable Algebra in Engineering, Communication and Computing, vol. 21, pp. 131-143, 2010. 